\section{PROTECTIVE INOCULATION AGAINST INFLUENZA.}

BY

W. H. WYNN, M.D., M.So., M.R.C.P.,

HONORARY PHYSICIAN TO THE GGNERAL HOSPITAL, BIRMFNGHAM: LECTORER IN GUINICAL MEDIGINE, ONIVERSITY OF BIRMINGHAM.

The possibility of a recrudescence of influenza and the distribution of vaccine by the Ministry of Hoalth has caused much questioning, both by medical men and the general public, as to the advisability of prophylactio inoculation. Although the true value of vaccines for prophylaxis against epidemic influenza cannot be established until the morbidity of a large sample of the population, inoculated before an outbreak, as compared with that in uninoculated controls, is submitted to expert statistical treatment, yet we possess much useful information gained during the last epidemics.

Until we know the true cause of influenza, the use of a vaccine must necessarily be empirical, but whatever the cause, there is little doubt that Pfeiffer's bacillus, and various strains of pneumococci and streptococci, are important secondary or coincident infeoting agents, and are responsible for the severe and fatal complications. There is evidence that a vaccine composed of these three organisms will not only diminish the mortality and reduce the complications of the disease, but will also lessen the incidence of uncomplicated influenza. If this latter fact can be proved, it must be taken seriously into account in any discussion upon the true virus of influenza. Most euthorities agree that a vaccine should contain several strains of Pfeiffer's bacillus, pneumococci and streptococci, but there is much difference of opinion as regards the proportions and number of these organisms to be used. Confronted with so many vaccines of varying strength and composition, the practitioner may well hesitate as to which he should choose.

The amount of immunity praduced by a vaccine depends within certain limits upon the size of the dose inoculated, the.limits probably being set not so much by the actual quantity of raccine used as by the amount of antigen which the tissues can set free from it. Experience with otlrer infections has taught as that when protection lasting for a considerable period is required large. doses of vaccine are necessary. Thus with typhoid fever doses of 1,000 and 2,000 million are used, and with pneumonia three injections at seven days' interval of 6,000 million pneumococei have been given. But in these two instances the vaccine is used upon selected individuals or communities and inocnlation of the general public is not undertaken. When, as with influenza, it is desirable to inoculate as many of the general public as possible, and sufficient immunity to protect during an epidemic period of a few month only is aimed at, such large doses may not be necessar $y$ It is also important to choose such a dose as will 'not produce unpleasant reactions. and so interfere with work and deter large numbers from being inoculated.

My. own preferemce is for a vaccine containing equal numbers of Pfeiffer's bacilli and various strains of preumo cocci and streptococei recently isolated. With pneumococci only first cultures are used, with streptococci either primary or first suboutbures. I am convinced that thie activity of a vaccine depends upon its being made from primary, or, at the most, first or second subcultures. Unfortunately it is difficult to make such vaccines on a large scale for widespread use, and the antigenic value of many commeroiel vaccines is greatly impaired. During the epidemic period of 1918-19 I gave a first dose of 100 million of each organism, and :after one week 200 million at first, but later increased to 200 and 400 million. These doses I am using at the present time. They seem sufficient to give adequate protection without causing more than a trifling reaction. In several cases these doses have been followed by a third of 800 million and even a fourth of 1,200 million. In my opinion when the increased protection of these larger doses is aimed at it is best to begin with a dose of $\mathbf{2 0 0}$ million, and give three or fourincreasing doses at weekly intervals, rather than to give two doses of 500 and 1,000 million.

OFer 1,000 persons were injected with two doses, about half having a maximum dose of 200 million and the remainder of 400 million. Of these there is a record of the result in 680. Of these only 14 developed influenza, but 4 of these were attacked three months after the last injection. In all cases the disease was mild, and there was no case of "pnetamonia. In one village in "which influenga wag very prezalent there were two large pre. paratory schools. In one no vaceine was used and nealy every boy, besides masters and servants, suffered from influenza. In the other sohool 75 boys and 38 adults were injected. One adult was -injected after infection had occurred and developed pneumonia All the others escaped influenza. In the village 31 adults and 16 children were injected, and all esoaped. One boy injeoted during the vacation returned to a boarding school, and 19 out of 23 boys suffered from influenza soon after the beginning of term; the inoculated boy was among the four:who esoaped. In another doetor's practice there were 35 carses of influenza, 7 with pneumonia; 40 immediate contaets with these patients were injected, and of these 3 had mild influenza, in 2 fever only lesting ono day. At one institution 117 nurses and maids out of a staff of 170 were inoculated during the autumn rof 1918. Of the $53 \mathrm{un}$ inoculated 25 had mild attacks in June, 1918, and escaped in the epidemies of Nopember and February. Of the 28 presurnably unprotected, 14 suffered from inflaenza, 2 with severe bronehopneumonia, of whom one died: Of 5 who only receive : orre inoculation one had mild imfinenza and one a severe attack: It is not pretended that these results have any statistical value or are free from fallacies, but they support the favourable results obtained elsewirere.

In America proteotive inoculation was practised on a much larger scale, and muoh larger doses of vacaine were given than in this country.

Rosenow ${ }^{1}$ used a vaceine with the formula: Preumococci, 3,000 million ('Type 1, 500 million; Type \&, 750 million; Type 3, 500 million; Type 4, 1,250 million); Streptococcus haemolytious, 1.000 million; B. influenzae, 500 mitlion: staph ylocoesi; 500 million, in $1 \mathrm{c.cm}$; 1 c.cm. was given as a tirst dose, and then 1 c.cm. and 1.5 c.cm. at intervals of seven days. The final doses, therefore, consisted of 7,500 million organisms. A vaccine of this formula is obtainable in this country. In my experience it is apt to cause rather unpleasant reactions, and Rosenow remarks thrat the numbar of severe reactions is sufficiently large to prevent general vaccination; except at the time of an acute emergency. It might be thong th that the injection of such large doses might have a $1 \mathbf{r}$-judicial effect upon chronic infections, especially of the respiratory tract. But the reports show that 951 persons with chronio bronchitis were besefted, and 38 made worse; 127 persons with chronic sinusitis were benelited, and 4 made worse; 121 with chronic myositis and 129 with chronio arthritis were benefited, and in one of the former and 22 of the latter the symptoms were aggravated; 997 pregnant women were inoculated, 'with excellent results.

In institutions where the condi ions among the vac. cinated and unvaccinated were comparable, 8,306 were injected three times and 9,388 were unvacinated. Among the vaccinated the incidence of influenza per $1,000 \mathrm{was} 31$ and among the unvaccinated 200 , the incidence of preumonia 1.0 and 12.0 per 1,000 respectively. Among tho general population 93,476 persons received three injections; the incidence of influenza was 87.9 per 1,000 , and of pneumonia 4.4 per 1,000, as compared with 281.8 and $21: 0$ per 1,000 among the unvaccinated. Taking all the results, the a verage mortality rate in the inoculated was one-fifth of that in the uninoculated.

Minaker and Irvine ${ }^{2}$ in San Francisco used a vaccine containing 5,000 million $B$. influenzae, 7,000 million pneumococci $(3,000$ million each of Type 1 and Type 2 , 1,000 million of Type 4), and 100 million Streptoooccus haemolyticus per cubic centimetre; $0.5,0.8$, and $1.0 \mathrm{c.cm}$. were given at three-day intervals. The final dose was therefore 12,100 million organisms. This, so far as I am aware, is the largest dose given for influenza. Of the civil population 1,080 were inoculated; their morbidity was 1.4 per cent. and mortality nil. These persons mixed with an uninoculated population whose morbidity was 5.3 per cent. and mortality 9.2 per cent.; 1,950 marines were inoculated and 8,232 not inoculated. The respeotive morbidity and mortality rates in the former were 1.8 and 2.8 per cent.; as compared with 15.7 and 5.0 in the datter. For uninoculated nurses and attendants in hospitals the morbidity was 33.8 per conto, among the inoculated 3.5 per cent. 
Cadman $^{3}$ in Winnipeg used a vaccine of 300 million streptococci, 200 million influenza bacilli, and 150 million pneumococci. Out of 7,600 soldiers, 4,842 were inoculated, but only about half received two doses. Of cases admitted to hospital, 6.05 per cent. of those previously inoculated developed pneumonia, against 17.1 in the uninoculated. The deaths in the uninoculated were 7.1 per cent., in the inoculated 1.7 per cent., but all these had only received one dose of vaccine; there were no deaths among those who had two injections. In the civil population 24,184 received two injections; the incidence of influenza was 9.8 per cent., pneumonia 0.57 per cent., and deatlıs 0.16 per cent., as compared with 21,285 uninoculated, among whom the incidence of influenza was 24.8 per cent., pneumonia 2.2 per cent., and deaths 0.66 per cent.

In England there has been an unnecessary timidity in the use of large doses for prophylaxis, and the original formula recommended by the War Office Conferencenamely, 60 million $B$. influenzae, 80 million streptococci, and 200 million pneumococci in 1 c.cm.-of which it was recommended that $0.5 \mathrm{c.cm}$. should be given as a first dose and 1 c.cm. after ten days, met with considerable criticism. Nevertheless the results, just published by Sir William Leishman, obtained in the Home Commands showed that this vaccine gave some measure of protection. Of 15,624 inoculated the incidence of influenza was 14.1 per 1,000 , of pulmonary complications 1.6 per 1,000 , and deaths 0.12 per 1,000 , as compared with 43,520 uninoculated giving an incidence of $47.3,13.3$, and 2.25 respectively. About half the inoculated received only one dose of the vaccine.

The vaccine now recommended and adopted by the Ministry of Health contains 400 million B. influenzae, 80 million streptococci, and 200 million pneumococci. Thus, whilst the dose of pneumococci and streptococci remains the same, the duse of influenza bacilli has been increased from 60 to 400 million. It would be interesting to have the reasons why this formula was adopted. The streptococcus is probably the most dangerous of the three organisms, and in view of Rosenow's experience with doses of 1,000 million of haemolytic streptococci, it seems advisable that as large a dose of this organism should be given as of the others. The experience of Lister, Cole, and others with pneumococcic vaccine shows that large doses can be given without undue reactions. With the increase of the dose of influenza bacilli to 400 million the point is made " that the strains employed should not have been so cultivated or so recently derived from cases as to be unduly toxic in their action." Some of the advantage of the larger dose is therefore lost by using less active cultures. The dose contrasts with that used in San Francisco, namely, 5,000 million influenza bacilli, with such excellent results.

The St. Mary's Hospital formula $-B$. influenzae 500 million, pneumococcus (mixed types) 1,000 million, and streptococcus 100 million, $\frac{1}{2}$ c.cm. to be followed by $1 \mathrm{c.cm}$. in a week-gives a larger dose and has been used on a large scale, but no results of its use have been published.

Of other vaccines that have been advocated Rosenow's vaccine suspended in oil instead of normal saline may, in the light of Fennel and Cecil and Vaughan's studies of the pneumococcic lipo-vaccine, give a greater degree of protection. Detoxicated vaccines are being strongly advertised. The evidence in their favour is not convincing. My own experience of them leads me to regard them as comparatively inert, and I doubt if any reliance should be placed upon them for purposes of prophylaxis.

There is evidence of a considerable degree of crossprotection from vaccines. In connexion with the severe outbreaks of camp septicaemia and bronchopneumonia in America, it was found that inoculation with a pneumo. coccic vaccine gave some protection against the streptococci responsible for the outbreals, the incidence of streptococcus pneumonia being nearly ten times as great among the unvaccinated as in those injected with the pneumococcic raccine. It must also be within the experience of many that during the epidemics of last winter, patients who were andergoing vaccine treatment for other complaints were comparatively immune to influenza. Also injection of the triple influenza vaccine often has a beneficial effect in patients suffering from chronic infections.

In the circular accompanying the vaccine issued by the Ministry of Health the inocalation of children under
3 years of age is not advised. It is difficult to under stand the reason of this. Young children show no specjal resistance to influenza, and their chance of recovery is less than in older patients. Children respond to vaccines very well, and I have had no hesitation in giving suitably reducei prophylactic doses to children under 3 years of age.

The records here given show that no medical man need hesitate to advise prophylactic injections to his patients. In most instances the vaccine of the Ministry of Healtl - will doubtless be used, but there is evidence that much larger doses can be safely used. Indeed, it seems advisable that persons particularly exposed to infection should reach larger doses, and $0.75 \mathrm{c.cm}$. and $1.5 \mathrm{c.cm}$. of the Ministry' vacoine or a vaccine of a stronger formula could be chosen It is to be hoped that in every case careful statistics will be kept, and that where the dose varies from that of the standard vaccine a note of this will be made.

1 Rosenow, Journ. Amor. REFERENCE8. Med. As80c. August 9th, 1919. p. 396 2. Minaker and Irvine, Journ. Amor. Med. Assoo. March 22nd, 1919 Madman, Lancet, May 24th, 1919 , p. 885. 14 Leishman, BRirisi Journ. Exper. Med., 1918, uxviii. p. 19.

\section{SOME UNUSUAL FORMS OF DYSENTERY.}

BY

GEORGE C. LOW, M.A., M.D., C.M., M.R.C.P.,

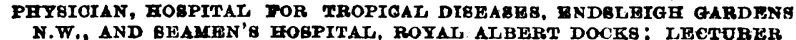
AND CONSOLTING PATHOLOGIST TO THE LONDON SCHOOL OP TROPIOAL MEDICTNE.

The term dysentery is more or less indiscriminately applied to any condition producing blood and mueus: in the stools, or even to diarrhoea alone.

For a proper classification the cause of the condition must be determined, and at the present day we talk of protozoal dysenteries, helminthio dysenteries, bacterial dysenteries, pseudo-dysenteries, and so on. As a general rule, when the term dysentery is used, unless some qualifying adjective is attached to it, either a case of amoebic or bacillary dysentery is indicated - namely, dysentery due to the Entamoeba histolytica or to the bacillus of Shiga or Flexner. We are apt to rest at this and consider the matter settled, but so many other con. ditions, many of them very common, may produce an almost similar clinical picture to these real dysenteries that a careful examination must always be made to exclude them before definitely settling our diagnosis.

A few specific instances culled from a large experience of the treatment of dysentery in London during the war will explain what I mean.

\section{Syphilis resembling Dysentery (Stphinitio} DYsentert).

Syphilitic ulceration of the rectum is not ancommon ultimately it leads to stricture of the bowel and other troubles. The patient passes bloody stools with mucus, and is at once branded as "dysentery" and probably dosed with emetine or given antidysenteric serum. I have the records of such a oase by me now :

The patient started his trouble in France, in 1916, with diarrhoea. Though the result of the examination of the stools was negative as to protozoa, the diagnosis of dysentery was mad and he was given emetine. He was no better but retarned to his work. He soon broke down again and spent the next year going in and out of various hospitals and having more emetine though again no protozos were found in his stools. At one o these hospitals a shadow was seen in the rectum on $x$-ray examination. Finally he came under my care in 1919. A sigmoidoscopic examination made by Sir James Cantlie then revealed ulceration with a stricture just below the sigmoil flexure; the opinion was that this was not malignant but sxphilitic. There was an old history of syphilis. The Wassermann reaction was negative. A course of novarsenobillou injections was then given and everything cleared up satis. factorily, the blood and mucus disappearing and the stools factorily, the blood and mucus disappearing and the stools
becoming normal in consistence and appearance. After some time he relapsed and a second series of injections was given. The condition again improved, but not so much as the first time. He is now on mercury and iodide. During his stay in hospital no protozoa were ever found in his stools.

\section{Malignant Disease.}

Mr. Dobell has told me of a case, very similar to the one described above, in which the patient was treated for dysentery and dosed heavily with emetine for quite a long 